\title{
Publisher's Note: Anisotropic Invariance and the Distribution of Quantum Correlations [Phys. Rev. Lett. 118, 010401 (2017)]
}

Shuming Cheng and Michael J. W. Hall

(Received 17 January 2017; published 2 February 2017)

DOI: 10.1103/PhysRevLett.118.059901

This paper was published online on 5 January 2017 with an error in the text on page 3 . On page 3 , the second line of the left-hand column should read as "lengths $a, b, c$ (or the concurrences) by the three-tangle $\tau \ldots$. ." The paper has been corrected as of 18 January 2017. The text is incorrect in the printed version of the journal. 